九州大学学術情報リポジトリ

Kyushu University Institutional Repository

\title{
Humanized Computational Intelligence with Interactive Evolutionary Computation
}

\section{高木，英行}

Department of Art and Information Design, Kyushu Institute of Design

http://hdl. handle. net/2324/4486305

出版情報：pp. 1-，2002-10-29

バージョン:

権利関係 : 


\title{
Humanized Computational Intelligence with Interactive Evolutionary Computation
}

\author{
Hideyuki Takagi \\ Kyushu Institute of Design (till Sept. 30, 2003) Kyushu University (since Oct. 1, 2003) \\ 4-9-1 Shiobaru, Minami-ku, Fukuoka, 815-8540 Japan \\ TEL\&FAX $+81-92-553-4555$ \\ takagi@kyushu-id.ac.jp (till Sept. 30, 2003) \\ takagi@design.kyushu-u.ac.jp (since Oct. 1, 2003)
}

\begin{abstract}
First, we show that human factors will become much more important for engineering in the future with concrete examples and propose the concept of humanized computational intelligence as one of the future research direction of computational intelligence research. Then, we introduce interactive evolutionary computation as a tool to realize the humanized computational intelligence. We also discuss several IEC researches in major fields.
\end{abstract}

\section{INTRODUCTION}

Computational intelligence in the 20th Century is characterized by the proposal of biologically inspired technologies and fusing these technologies. All major seed technologies of computational intelligence were presented by the 1960's. The basis of neural networks (NNs) was established by the perceptron model in 1958 following a neuron model in 1943. Fuzzy sets in 1965 became the basis of fuzzy systems (FS) and led to several FS applications following the first application of fuzzy control in 1973. Following the independent proposal of evolutionary programming (EP), evolutionary strategy (ES), and genetic algorithms (GAs) in the 1960's and 1970's, evolutionary computation (EC) came to form a group of useful techniques: EP, ES, GA, and genetic programming (GP).

Practical applications of each computational intelligence technology became popular since the late 1980 's, and many $\mathrm{NNs}, \mathrm{FS}$, and EC systems were developed for industrial and consumer products. Cooperative or fusion technologies of NNs, FS, and EC began in the late 1980's. Their applications have spread to the industrial and consumer products in the 1990 's, too [1].

As we glance over the computational intelligence research in the last several decades, NNs, FS, and EC have played a main role, and their fusion technologies have become practical. How should the computational intelligence research expand now? We discuss this point in section II and believe that the concept of humanized computational intelligence will be one of its answers. Finally, we introduce an interactive evolutionary computation (IEC) as one of technologies realizing the concept in section III.

\section{HUMANIZED COMPUTATIONAL INTELLIGENCE}

We saw the feature of computational intelligence in the 20th Century in the last section. Then, what characterizes computational intelligence from this point forward?

We believe that one of several research directions of this technology is to embed directly the capability of a human being or living things into computational intelligence, instead of their models. Let us call this type of technology as humanized computational intelligence. The background of this view is: (1) any technology or system has advantages and disadvantages, and the combination of these advantages is expected to be more powerful than the original advantages, (2) humans can offer performance that is superior to models of that performance, and (3) conventional computational intelligence can not handle some real-world tasks that need human evaluation, such as visual or auditory inspection at the final process of manufacturing or human preference.

Let's see some examples of a humanized technology shown in Fig. 1.

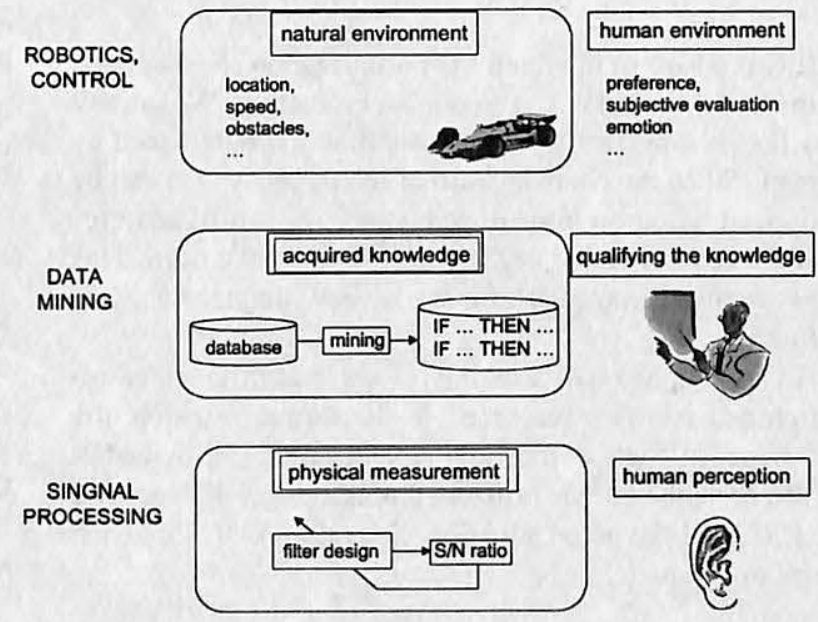

Fig. 1: Humanized technology for conventional engineering tasks.

A robot controller inputs robot's position, speed, and distance to obstacles from its physical environment and use them as control

information. Unlike industrial robots, a natural behavior that matches human preference or lets humans feel safe and comfortable is required for consumer robots. To deal with such a human environment, a human must be involved in the robot control system.

Data mining is a technology to acquire knowledge from numerical data. We can acquire rules automatically from data by using AI tools, neuro-fuzzy systems, or EC-fuzzy systems. However, if the purpose is to consider the quality of rules and obtain better rules, a domain expert who can evaluate which rule is better and how must be involved in the knowledge acquisition systems. 
If our purpose of using signal processing technology is to handle physical indexes such as signal-noise ratio, we may use conventional mathematical-based signal processing methods. But, if the purpose is to make a signal auditorily or visually better, a human who finally judges should be involved in the signal processing system rather than using perceptual evaluation models.

It is need for humanized computational intelligence to make a framework to combine human factors, especially preference, psychological aspect, or KANSE in general, with computational intelligence. Humans have two types of capabilities; one is a knowledge aspect, which deals with reasoning, knowledge expression, knowledge acquisition, associated memory, and learning, and another is an aspect of $K A N S E I$, which is a generic term of intuition, preference, subjective evaluation, feeling, and other psychological aspects. We need a frameworks and methodology to deal with the $K A N S E I$ aspect from an engineering perspective.

There are several possible approaches to realize humanized computational intelligence. Interactive evolutionary computation (IEC) is one of them. Let us see the IEC in the following section.

\section{INTERACTIVE EVOLUTIONARY COMPUTATION}

\section{A. IEC as a framework for Humanized Computational Intel- ligence}

IEC is a system in which EC optimizes the characteristic of the target system based on human evaluation. We can say that IEC is a system whose fitness function is replaced by human. Since the characteristic of the target system can be optimized based on human evaluation, we can directly reflect our sense of value or KANSEI into system design. This is a convenient framework for humanized computational intelligence.

IEC research consists mainly of application-oriented research and interface research. In the former research, the IEC has been applied to several varieties of fields. In the latter research, the fatigue problem that is a major drawback of the IEC has been given attention. See reference [2] for each research in detail.

\section{B. IEC Application Research}

IEC application fields mainly includes artistic field, engineering field, and education, game, and other fields [2].

The aim of IEC-based artistic applications is to create computer graphics or music based on user's sense of art but not on user's skill. Unlike conventional artistic design, this approach does not require skills of computer graphic or playing music instruments. Some applications in this field include graphic arts and animation, 3-D computer graphics lighting, music, editorial design, industrial design, and facial image generation.

There are two types of engineering applications. One is visual- or auditory-based signal processing which optimizes signal processing filters based on human visual or auditory inspection. Another is polishing up the target system by embedding knowledge or know-how of domain experts. Some application in this field includes speech processing and synthesis, hearing-aid fitting, virtual reality, media database retrieval, data mining, image processing, control and robotics, food industry, and geophysics.

Other application field includes artistic education, entertainment such as games, network forum to create better idea, therapy, and other.

\section{IEC Interface Research}

IEC has some problems that the normal EC does not have. The biggest remaining problem is human fatigue which is caused by the IEC framework that a human user must cooperate with a tireless computer and evaluate EC individuals. The second problem is how to search for a goal with a smaller population size within a fewer number of searching generations. This requirement is inevitable due to the fatigue problem. The third problem is how to let an IEC user evaluate time-variant individuals such as sounds or movies that cannot be compared spatially with less fatigue and less operation time. IEC interface research has been conducted to solve these problems and to make IEC more practical.

The IEC interface research conducted so far includes: a discrete fitness value input method to reduce psychological fatigue, the prediction of fitness values that predicts the characteristics of human evaluation to increase real number of individuals for the EC (not for the user) and support human evaluation, IEC interface for time-sequential display tasks such as sounds or movies, EC acceleration especially in early generations, and active intervention that allow users to join the EC search in addition to subjective evaluation in order to reduce user's psychological fatigue.

\section{CONCLUSION}

The future direction of computational intelligence has been discussed through surveying past research and emphasizing that the concept of humanized computational intelligence is one of keywords to pilot the future of computational intelligence. IEC is one of key technologies for developing this concept. As seen in this paper, several new approaches based on IEC have started by combining human capabilities and show the high potential of IEC. We hope that this concept emphasized in this paper becomes a good guidepost to those who work in this field.

[1] Hideyuki Takagi, "Fusion Technology of Neural Networks and Fuzzy Systems: A Chronicled Progression from the Laboratory to Our Daily Lives," Int. J. of Applied Mathematics and Computer Science, 10(4), pp.647-673 (2000).

[2] Hideyuki Takagi, "Interactive Evolutionary Computation: Fusion of the Capacities of EC Optimization and Human Evaluation," Proceedings of the IEEE, 89(9), pp.1275-1296 (2001). 